\title{
Mapeamento da "Covid longa" em pacientes assistidos pela USF Itamarati e previamente infectados por SARS-CoV-2
}

Mapping of "Long Covid" in patients assisted by the USF Itamarati and previously infected with SARS-CoV-2

Mapeo de "Long Covid" en pacientes asistidos por la USF Itamarati y previamente infectados con SARS-CoV-2

\section{Resumo}

Introdução: Uma fração dos pacientes com COVID-19 que passaram por uma fase sintomática aguda variável da doença está apresentando efeitos contínuos da doença, com queixas como névoa mental, períodos latentes retardados na lembrança de eventos do passado recente, taquicardia, fadiga extrema, incapacidade de realizar tarefas físicas diárias, para citar alguns dentre uma série complexa de sinais e sintomas. Objetivo: estimar e tabular as principais sequelas e os sintomas presentes nos pacientes assistidos pela USF Itamarati infectados por SARSCoV-2 durante o período compreendido entre 01/04/2020 e 01/10/2021. Metodologia: abordagem observacional analítica desenvolvido na Unidade Básica de Saúde Itamarati, localizada no bairro Jardim Itamarati, na cidade de Patos de Minas, Minas Gerais, sob dados da Plataforma Viver, prontuários físicos e DATASUS. Resultados: a prevalência de sintoma persistente foi, principalmente, a cefaleia, seguida pela dispneia, eflúvio telógeno e fadiga, respectivamente. Além disso, constatou-se, neste estudo, que a média de incidência dessas queixas, quando associadas ao tempo da positividade para COVID-19, foi de, aproximadamente, dentre 3 e 6 meses. Considerações finais: as sequelas da COVID-19 podem afetar a qualidade de vida e até ameaçar a vida. Cada paciente que foi infectado com a COVID-19 pode apresentar sintomas como fadiga, falta de ar, dores de cabeça, dores musculares, queda de cabelo, perda de paladar e olfato, dor no peito, tontura, tromboses, palpitações, depressão e ansiedade, perda de raciocínio e memória. Sendo assim, uma avaliação individualizada por uma equipe multiprofissional é essencial para planejar todo o processo de recuperação do corpo e também o acompanhamento de órgãos essenciais, como coração, pulmões e músculos, para minimizar as chances de eventos cardiovasculares e trombóticos além de sequelas pulmonares, aumento de morbidade e baixa qualidade de vida por desnutrição, fraqueza muscular e dores.

Palavras-chave: Covid longa; COVID-19; Sintomas; Sequelas. 


\begin{abstract}
Introduction: A fraction of patients with COVID-19 who have gone through a variable acute symptomatic phase of the disease are experiencing ongoing effects of the disease, with complaints such as mental fog, delayed latent periods in remembering events from the recent past, tachycardia, extreme fatigue, disability of performing daily physical tasks, to name a few among a complex array of signs and symptoms. Objective: To estimate and tabulate the main sequelae and symptoms present in patients assisted by the USF Itamarati infected with SARS-CoV-2 during the period between 01/04/2020 and 01/10/2021. Methodology: analytical observational approach developed at the Itamarati Basic Health Unit, located in the Jardim Itamarati neighborhood, in the city of Patos de Minas, Minas Gerais, using data from the Viver Platform, physical records and DATASUS. Results: the prevalence of persistent symptoms was mainly headache, followed by dyspnea, telogen effluvium and fatigue, respectively. In addition, it was found in this study that the mean incidence of these complaints, when associated with the time of positivity for COVID-19, was approximately between 3 and 6 months. Final considerations: the sequelae of COVID-19 can affect the quality of life and even threaten life. Every patient who has been infected with COVID19 may experience symptoms such as fatigue, shortness of breath, headaches, muscle aches, hair loss, loss of taste and smell, chest pain, dizziness, strokes, palpitations, depression and anxiety, loss of reasoning and memory. Therefore, an individualized assessment by a multidisciplinary team is essential to plan the entire body recovery process and also the monitoring of essential organs, such as the heart, lungs and muscles, to minimize the chances of cardiovascular and thrombotic events in addition to pulmonary sequelae, increased morbidity and poor quality of life due to malnutrition, muscle weakness and pain.
\end{abstract}

Keywords: Long Covid; COVID-19; Symptoms; Sequelae.

\begin{abstract}
Resumen
Introducción: Una fracción de los pacientes con COVID-19 que han pasado por una fase sintomática aguda variable de la enfermedad están experimentando efectos continuos de la enfermedad, con quejas como niebla mental, retrasos en los períodos latentes para recordar eventos del pasado reciente, taquicardia, fatiga extrema, incapacidad para realizar las tareas físicas diarias, por nombrar algunos entre una compleja gama de signos y síntomas. Objetivo: Estimar y tabular las principales secuelas y síntomas presentes en pacientes atendidos por la USF Itamarati infectados con SARS-CoV-2 durante el período comprendido entre el 01/04/2020 y el 01/10/2021. Metodología: enfoque observacional analítico desarrollado en la Unidad Básica de Salud Itamarati, ubicada en el barrio Jardim Itamarati, en la ciudad de Patos de Minas, Minas Gerais, utilizando datos de la Plataforma Viver, registros físicos y DATASUS. Resultados: la prevalencia de síntomas persistentes fue principalmente cefalea, seguida de disnea, efluvio telógeno y fatiga, respectivamente. Además, se encontró en este estudio que la incidencia media de estas quejas, cuando se asocian con el tiempo de positividad para COVID-19, fue aproximadamente entre 3 y 6 meses. Consideraciones finales: las secuelas de COVID-19 pueden afectar la calidad de vida e incluso amenazarla. Todo paciente que ha sido infectado con COVID-19 puede experimentar síntomas como fatiga, dificultad para respirar, dolores de cabeza, dolores musculares, pérdida de cabello, pérdida del gusto y del olfato, dolor de pecho, mareos, accidentes cerebrovasculares, palpitaciones, depresión y ansiedad, pérdida de razonamiento y memoria. Por ello, una valoración individualizada por parte de un equipo multidisciplinar es fundamental para planificar todo el proceso de recuperación corporal y también el seguimiento de órganos esenciales, como corazón, pulmones y músculos, para minimizar las posibilidades de eventos cardiovasculares y trombóticos además de secuelas pulmonares. aumento de la morbilidad y mala calidad de vida debido a la desnutrición, debilidad muscular y dolor.
\end{abstract}

Palabras clave: Covid largo; COVID-19; Síntomas; Secuelas.

\title{
1. Introdução
}

Uma fração dos pacientes com COVID-19 que passaram por uma fase sintomática aguda variável da doença está apresentando efeitos contínuos da doença, com queixas como névoa mental, períodos latentes retardados na lembrança de eventos do passado recente, taquicardia, fadiga extrema, incapacidade de realizar tarefas físicas diárias, para citar alguns dentre uma série complexa de sinais e sintomas. Os sinais e sintomas são tão diversos e relacionados a vários órgãos e sistemas que é difícil atribuir a eles uma terminologia adequada. Na tentativa de reconhecer este estado único de desconforto e incapacidade relacionados ao COVID-19, é necessário fornecer um termo melhor para esse estado sindrômico (Greenhalg et al., 2020).

Sendo assim, "Covid longa" é um termo usado para descrever a doença em pessoas que se recuperaram da COVID19, mas ainda relatam efeitos duradouros da infecção ou apresentam os sintomas usuais há muito mais tempo do que o esperado. Muitas pessoas, incluindo médicos infectados, compartilharam suas experiências anedóticas nas redes sociais, na mídia tradicional e por meio de grupos de pacientes (Puntmann et al., 2020). Além de evidências anedóticas, ainda há poucas 
pesquisas sobre o assunto. No entanto, está sendo ativamente discutido na comunidade de pesquisa. Além disso, a ocorrência de complicações multiorgânicas não é inesperada, haja vista que o receptor de entrada ACE2 da SARS-CoV-2 é expresso em vários tecidos (Zubair et al., 2020). Entre as possíveis sequelas, apontam-se as neurológicas, respiratórias, psicológicas, vasculares, gastrointestinais, cutâneas e até mesmo as relacionadas ao olfato e paladar, bem como os principais sintomas apresentados no pós-Covid comumente relatados são cefaleia, tontura, náusea, confusão mental, mialgia, distúrbios vasculares, bem como algumas consequências graves, como hemorragia intracerebral aguda, trombose de seio venoso cerebral, encefalopatia e síndromes autoimunes (Zhao et al., 2020).

Desse modo, é necessário que os profissionais de saúde ouçam os pacientes para entender suas preocupações, validem suas experiências e gerenciem seus sintomas e comorbidades, encaminhando os pacientes quando necessário, haja vista que muitos deles já se sentem rejeitados ou esquecidos. Sem definições clínicas claras da "Covid longa" e na ausência de um teste diagnóstico ou de um tratamento eficaz, os profissionais de saúde encontram-se em uma posição difícil para ajudar seus pacientes (Yelin et al., 2020). Por isso, o conhecimento de evolução lenta de outras condições mal compreendidas (como dor crônica e distúrbios funcionais) mostra os riscos para os pacientes que sentem que seus sintomas estão sendo diminuídos ou ignorados. Sem reconhecimento claro, comunicação honesta e pesquisa cuidadosa centrada no paciente, os pacientes enfrentam resultados insatisfatórios (Rayner et al., 2020).

Por isso, este projeto justifica-se pelo fato de que a COVID-19 emergiu preocupações globais, não apenas na fase aguda da doença e suas consequências imediatas, mas também eventuais sequelas a médio e longo prazo, aliado ao risco de cronicidade de alguns sintomas. Por isso, ao analisar e tabular os principais sintomas persistentes corroborados pela doença, o projeto apresenta-se com suma valia para a comunidade científica, com intuito de gerir recursos para esses pacientes, bem como de traçar estratégias propedêuticas e terapêuticas para lidar com essas sequelas duradouras. Assim, o objetivo deste estudo foi estimar e tabular as principais sequelas e os sintomas presentes nos pacientes assistidos pela USF Itamarati infectados por SARS-CoV-2 durante o período compreendido entre 01/04/2020 e 01/10/2021.

\section{Metodologia}

O presente estudo é pautado em uma abordagem observacional analítica desenvolvido na Unidade Básica de Saúde Itamarati, localizada no bairro Jardim Itamarati, na cidade de Patos de Minas, com o objetivo de estabelecer e tabular quais são as principais sequelas e sintomas relatados por pacientes pós infectados, por pelo menos um mês, por SARS-CoV-2. A pesquisa foi realizada na USF Itamarati, por meio da Plataforma Viver e, posteriormente, por meio de análise individual e detalhada de prontuários físicos que estão presentes na Instituição. Os participantes incluídos no estudo foram todos aqueles cadastrados, sob atendimento na USF, com o CID-10 Z20 (COVID-19 confirmada) em seus prontuários, no período compreendido entre 01/04/2020 e 01/10/2021. Os pacientes cujo prontuários não estejam acessíveis, que não estejam residindo em localidades não atribuíveis a UBS Itamarati ou que faleceram foram excluídos da amostra que será estudada. Assim, a amostra totalizou 55 pacientes. Para o seguinte estudo, pretendeu-se coletar os números de prontuário eletrônico dos pacientes cujo CID-10 Z20 estivessem inclusos, no período correspondente de 18 meses. Após a coleta, foram avaliados, detalhadamente, os prontuários físicos dos respectivos pacientes.

Este projeto foi submetido ao Comitê de Ética em Pesquisa do Centro Universitário de Patos de Minas via Plataforma Brasil, sob número 4.982.290. O mesmo obedeceu a legislação nacional vigente, para realização da pesquisa envolvendo seres humanos, conforme a Resolução do Conselho Nacional de Saúde 466/2012. O presente estudo contou com o aporte de trabalhos acadêmicos entre os anos de 2020 e 2021, por meio do acesso às principais bases de dados, a saber: dados National Library of Medicine (PubMed MEDLINE), Scientific Electronic Library Online (Scielo), Cochrane Database of Systematic 
Reviews (CDSR), Google Scholar, Biblioteca Virtual em Saúde (BVS) e EBSCO Information Services, no período compreendido entre outubro e novembro de 2021.

\section{Resultados e Discussão}

Para a disposição dos dados, foram avaliados, essencialmente, o número total de pacientes cujo CID-10 Z20 (COVID19 confirmada) foi inserido no prontuário, e que estão atualmente cadastrados na unidade em questão. Conforme disposto na tabela 1, a USF do bairro Itamarati abrange um total de 55 pacientes acometidos por COVID-19 cadastrados conforme CID-10 Z20, sendo estes representados por 16 homens e 39 mulheres, respectivamente, $29 \%$ e 71\%, em que é possível observar uma prevalência geral de mulheres infectaas. Além disso, conforme exposto na tabela 2 , a média de idade do perfil do paciente obeso é entre os 40 e 60 anos, o que ilustra a maior prevalência dessa patologia na idade adulta. Os dados referentes ao perfil do paciente obeso cadastrado atualmente na USF Itamarati, entre as idades 10-77 anos, foram coletados na Plataforma Viver, no período estipulado de 18 meses, e dispostos nas seguintes tabelas:

Tabela 1: Número total de pacientes da amostra divididos por sexo.

\begin{tabular}{ccc}
\hline Sexo & fa & fr (\%) \\
\hline Masculino & 16 & $29 \%$ \\
Feminino & 39 & $71 \%$ \\
Total & 55 & $100 \%$ \\
\hline
\end{tabular}

Fonte: Autores (2021).

Tabela 2: Número total de pacientes da amostra divididos por idade.

\begin{tabular}{c|ccc}
\hline \multicolumn{2}{c}{ Idade } & fa & fr (\%) \\
\hline 10 & ----20 & 4 & $7 \%$ \\
20 & ----30 & 4 & $7 \%$ \\
30 & ----40 & 11 & $20 \%$ \\
40 & ----50 & 13 & $24 \%$ \\
50 & ----60 & 14 & $26 \%$ \\
60 & ----70 & 6 & $11 \%$ \\
70 & ----80 & 3 & $5 \%$ \\
\multicolumn{2}{c}{ Total } & 55 & $100 \%$ \\
\hline
\end{tabular}

Fonte: Autores (2021).

Nota-se que a prevalência de sintoma persistente foi, principalmente, a cefaleia, seguida pela dispneia, eflúvio telógeno e fadiga, respectivamente, conforme elucidado pela Tabela 3 e Figura 1. Além disso, constatou-se, neste estudo, que a média de incidência dessas queixas, quando associadas ao tempo da positividade para COVID-19, foi de, aproximadamente, dentre 3 e 6 meses.

Tabela 3: Incidência de sintomas pós-COVID pela amostra.

\begin{tabular}{ccc}
\hline Sintomas & fa & fr \\
\hline Fadiga & 23 & $41,8 \%$ \\
Dispneia & 39 & $70,9 \%$ \\
Cefaleia & 43 & $78,1 \%$ \\
Anosmia persistente & 6 & $10,9 \%$ \\
Comprometimento cognitivo & 4 & $7,3 \%$ \\
Eflúvio telógeno & 24 & $43,6 \%$ \\
Dificuldade de concentração e sonolência & 9 & $16,3 \%$ \\
\hline
\end{tabular}

Fonte: Autores (2021). 
Figura 1: Incidência de sintomas pós-COVID pela amostra.

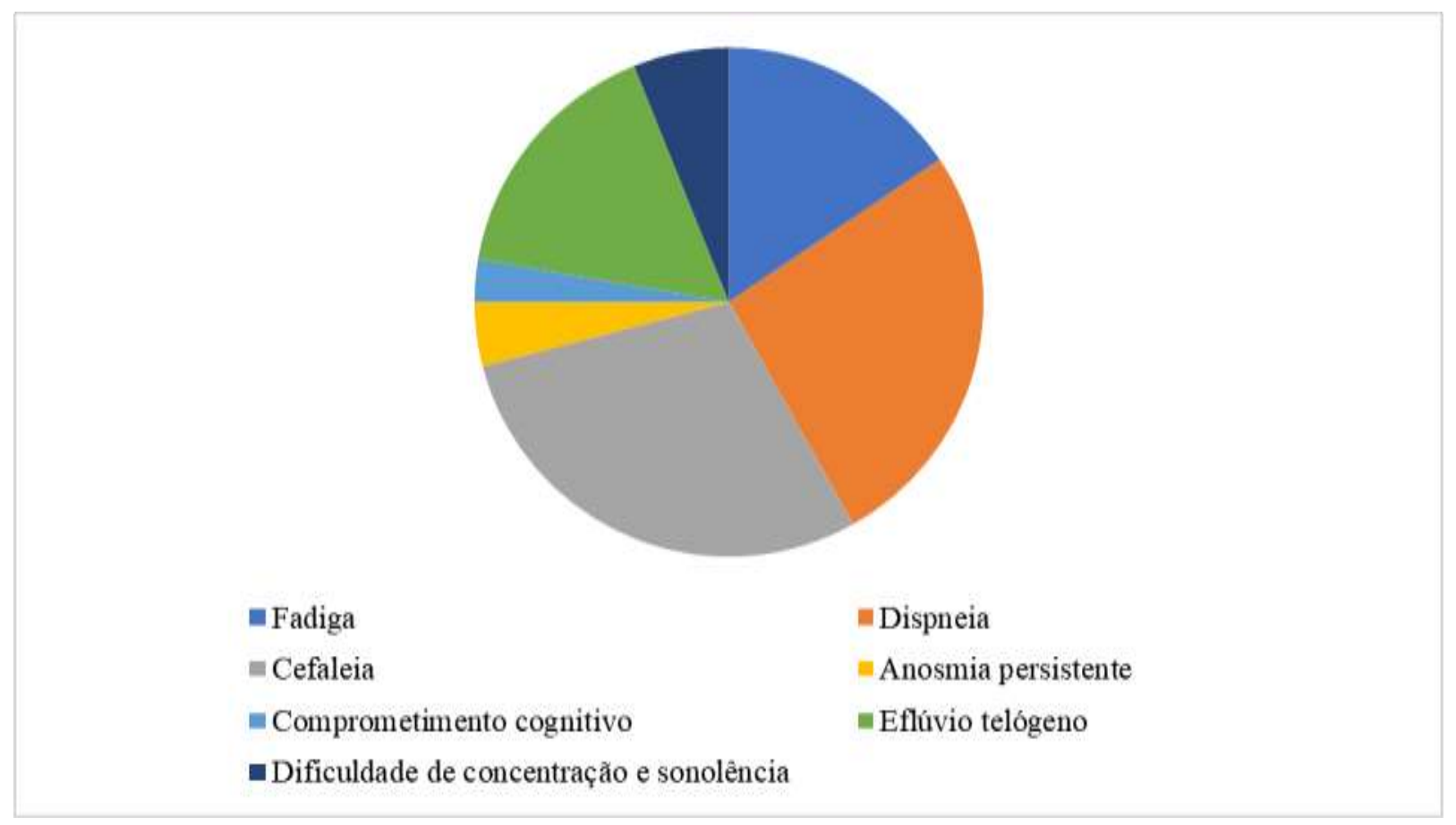

Fonte: Autores (2021).

Aliado a esses achados, o conjunto de sintomas inespecíficos vem sendo chamado, por especialistas, de "Síndrome pós-Covid" ou "Covid longa", como algo que acomete, e não apenas os pacientes graves que necessitaram de tratamento hospitalar e que passaram por longos períodos de internação em Unidades de Terapia Intensiva (UTIs), pois nota-se a quantidade de relatos de pacientes que apresentaram a forma branda ou moderada da doença também continuaram com algum tipo de sequela persistente (Zhao et al., 2020).

Entre os sintomas mais frequentes observados em análises clínicas e com base nos relatos dos pacientes estão, além da perda de olfato e paladar, dores musculares e nas articulações, fadiga, taquicardia, hipertensão ou hipotensão sem causa determinada e ainda dispneia (desconforto respiratório que pode se manifestar de diferentes maneiras em sensações como falta de ar ou aperto no peito) (Daniel et al., 2021). No entanto, não se sabe afirmar, por exemplo, exatamente, porque ocorrem as complicações extrapulmonares, por quanto tempo irão persistir e que consequências a médio e longo prazos podem trazer (Rayner et al., 2020).

É certo que a experiência com os sintomas prolongados da COVID-19 pode variar completamente de uma pessoa para outra. Há pacientes que se queixam de comprometimento cognitivo aliado à perda de memória e dificuldade de concentração, após o contato com o vírus SARS-CoV-2, e também relatos que se encaixam naquilo que no jargão técnico conhece por "disautonomia", um transtorno provocado por alterações do sistema nervoso autônomo que pode afetar o funcionamento do coração, bexiga e intestino, entre outros órgãos (Candan et al., 2020). Principalmente no caso de pacientes graves, que permaneceram muito tempo em isolamento durante a internação, sem possibilidade de contato com familiares e com visitas limitadas por barreiras físicas, podem desenvolver uma síndrome pós-traumática (Puntmann et al., 2020).

Segundo estudo de Carfi et al. (2020), de um total de 143 pacientes avaliados na Itália mais de dois meses depois de terem alta, apenas 18 estavam completamente livres de qualquer sintoma relacionado a COVID-19, enquanto 87,4\% relataram persistência de pelo menos um sintoma, entre eles fadiga $(53,1 \%)$ e falta de ar (43,4\%). Dor nas articulações foi observada em $27,3 \%$ e dor no peito, em $21,7 \%$. Além disso, $44,1 \%$ dos pacientes apresentaram piora da qualidade de vida. O detalhe é que 
apenas 12,6\% haviam tido a forma aguda da doença com passagem por uma UTI, e, nesses casos, é previsível que as sequelas permaneçam por um período mais longo enquanto dura a reabilitação.

\section{Considerações Finais}

As sequelas da COVID-19 podem afetar a qualidade de vida e até ameaçar a vida. Cada paciente que foi infectado com a COVID-19 pode apresentar sintomas como fadiga, falta de ar, dores de cabeça, dores musculares, queda de cabelo, perda de paladar e olfato, dor no peito, tontura, tromboses, palpitações, depressão e ansiedade, perda de raciocínio e memória. Sendo assim, uma avaliação individualizada por uma equipe multiprofissional é essencial para planejar todo o processo de recuperação do corpo e também o acompanhamento de órgãos essenciais, como coração, pulmões e músculos, para minimizar as chances de eventos cardiovasculares e trombóticos além de sequelas pulmonares, aumento de morbidade e baixa qualidade de vida por desnutrição, fraqueza muscular e dores.

\section{Referências}

Candan, S. A., et al. (2020). Consideration of prevention and management of long-term consequences of post-acute respiratory distress syndrome in patients with COVID-19. Physiother Theory Pract., 36 (6), 663-668.

Carfi, A., et al. (2020). Sintomas persistentes em pacientes após COVID-19 agudo. JAMA, 324 (6), 603-605.

Daniel, C. R., et al. (2021). Estamos olhando para os indivíduos pós-COVID como deveríamos? Journals Bahiana, 10 (4), 588-590.

Greenhalg, T., et al. (2020). Manejo de Covid-19 pós-agudo na atenção primária. The BMJ, 370 (3), 1-9.

Puntmann, V. O., et al. (2020). Resultados da ressonância magnética cardiovascular em pacientes recentemente recuperados da doença coronavírus 2019 (COVID-19). JAMA Cardiology, 28 (5), 78-85.

Rajpal, S., et al. (2020). Achados de ressonância magnética cardiovascular em atletas competitivos em recuperação de infecção por COVID-19. JAMA Cardiology, 31 (6), 1-8.

Rayner, C., et al. (2020). COVID-19: o curso prolongado e recorrente da doença tem implicações para o retorno de trabalhadores. The BMJ, 5 (7), 13-19.

Yelin, D., et al. (2020). Consequências de longo prazo do COVID-19: necessidades de pesquisa. The Lancet Infectious Diseases, 20 (10), $1115-1117$.

Zhao, Y. M., et al. (2020). Estudo de acompanhamento da função pulmonar e características fisiológicas relacionadas de sobreviventes de COVID-19 três meses após a recuperação. Clinical Medicine, 25 (6), 63-70.

Zubair, A. S., et al. (2020). Neuropatogênese e manifestações neurológicas dos coronavírus na era da doença coronavírus 2019: uma revisão. JAMA Neurology, 77 (8), 1018-1027. 